\title{
Vital Signs Date Time of Reference Timepoint
}

National Cancer Institute

\section{Source}

National Cancer Institute. Vital Signs Date Time of Reference Timepoint. NCI Thesaurus.

Code C88011.

The date and time of a vital signs reference point. 